\title{
Plasma Homovanillic Acid and Performance on Motor and Cognitive Tasks in Community-Dwelling Elderly: MacArthur Studies of Successful Aging
}

Faroog Amin, M.D., Teresa E. Seeman, Ph.D., Richard C. Mohs, Ph.D., Michael Davidson, M.D., Peter Knott, Ph.D., Lisa F. Berkman, Ph.D., Marilyn Albert, Ph.D., and Dan Blazer, M.D., Ph.D.

To investigate the dopaminergic correlates of the agingrelated motor and cognitive deficits, the dopamine metabolite homovanillic acid (HVA) in plasma was studied in a community-dwelling elderly cohort $(\mathrm{n}=$ 141). The results showed that hand-signature time (HST), a measure of bradykinesia, correlated negatively with plasma HVA $(\mathrm{r}=-0.24, \mathrm{p}<.007)$. Similarities task performance showed a trend-level positive correlation with plasma HVA $(\mathrm{r}=0.15, \mathrm{p}=.08)$. Because plasma HVA is derived from several sources including central dopaminergic neurons and both central and peripheral noradrenergic neurons, the noradrenergic metabolite 3-methoxy-4-hydroxyphenylglycol (MHPG) in plasma was also measured to indirectly estimate the relationships, with HVA originating from noradrenergic metabolism. Plasma MHPG significantly correlated with similarities scores $(\mathrm{r}=0.34, \mathrm{p}<.001)$ but not with HST. The results suggested that the association of HVA with prolonged HST may be related to central dopamine metabolism, but its association with similarities scores may be due to noradrenergic metabolism. The results raise the possibility that prolonged HST may be an indicator of preclinical brain dopamine loss in the elderly. [Neuropsychopharmacology 10:29-35, 1994]
KEY WORDS: Dopamine; Homovanillic acid; Bradykinesia; Aging; Plasma; Striatum; Brain

Aging is known to be associated with a loss of brain dopamine (DA) (Carlsson and Winblad 1976; McGeer et al. 1977; Hornykiewicz et al. 1988; Wallin and Gott-

From the Bronx VA Medical Center, Mount Sinai School of Medicine, Department of Psychiatry (FA, RCM, MD, PK), New York, New York; Yale University School of Medicine, Department of Epidemiology and Public Health (TES, LFB), New Haven, Connecticut; Massachusetts General Hospital, Department of Psychiatry (MA), Boston, Massachusetts; and Duke University Medical Center, Department of Psychiatry (DB), Durham, North Carolina.

Address correspondence to: Farooq Amin, M.D., Psychiatry Service (116-A), Bronx VA Medical Center, 130 West Kingsbridge Road, Bronx, New York 10468.

Received April 13, 1993; revised August 25, 1993; accepted August 28,1993 . fries 1990). Although this loss of brain DA is not severe enough in most elderly to cause characteristic disorders of DA deficiency such as Parkinson's disease, varying degrees of subclinical DA losses are probably present in the elderly that may contribute to their aging-related deterioration in motor and cognitive functions. To identify the dopaminergic correlates of certain motor and cognitive tasks, the major DA metabolite homovanillic acid (HVA) in plasma was studied in a communitydwelling elderly cohort. Several lines of evidence suggest that plasma HVA is derived in part from the brain DA neurons and that plasma-HVA concentrations under controlled conditions may reflect central DA activity (Bacopoulos et al. 1979; Kendler et al. 1982, 1984; Konicki et al. 1991; Amin et al. 1992). Because bradykinesia and gait disturbances are common symptoms 
of Parkinson's disease, hand-signature time (HST) and walking time were selected as the motor measures of interest. It was hypothesized that plasma HVA would negatively correlate with prolonged HST and walking time. It was also hypothesized that plasma HVA would correlate with performance on the similarities task, which is a measure of abstraction that is impaired by frontotemporal lesions (McFie 1975) and may be associated with the central DA activity (Loranger et al. 1972; Stern and Langston 1985).

\section{SUBJECTS AND METHODS}

\section{Subject Population}

Subjects were selected from three larger samples of elderly individuals (from Durham, NC; East Boston, MA; and New Haven, CT) participating in the MacArthur Foundation Network on Successful Aging MultiSite study of community-dwelling elderly (Berkman et al. 1993). The MacArthur study sample was in turn derived from the population-based samples of individuals who are part of the National Institute on Aging funded Established Populations for Epidemiologic Studies of the Elderly (EPESE) (Cornoni-Huntley et al. 1986). Age range of the MacArthur study sample had been restricted to 70 to 79 years at the time of their 1988 EPESE interview to minimize the effect of age on results. The EPESE subjects had been screened for inclusion into the MacArthur study sample on the basis of physical and cognitive function criteria to identify a cohort with upper $30 \%$ of functional ability, that is, "successful aging" subjects $(n=1,192)$. In addition, smaller numbers of age- and sex-matched subjects from the lower tertile ( $n=82$ with "low functioning") and the middle tertile ( $n=80$ with "medium functioning") had also been included in the MacArthur sample (Berkman et al. 1993).

As part of the MacArthur study these individuals were interviewed in their homes for the assessment of motor and cognitive performance, psychosocial characteristics, and state of health (Berkman et al. 1993). A complete description of the assessment battery is included in Berkman et al. (1993) and Inouye et al. (1993). Hand-signature time, walking time, similarities task performances, and blood samples were obtained as part of these home visits. The subjects for this study were selected from the MacArthur cohort if they did not fall into one or more of the following categories. This was done to minimize the major confounding influences on plasma HVA. (1) Subjects reporting any known psychiatric or neurological disease. (2) Subjects who reported taking drugs known to affect HVA measurements: that is, antipsychotic, antidepressant, antiparkinsonian, beta-blockers, indomethacin, antiepileptic, diuretic, and sleeping drugs. (3) Subjects who had eaten within 8 hours of testing. One hundred forty-seven subjects were eligible for this study; plasma samples were not available for four subjects, and two additional subjects were later excluded due to errors in their data.

As part of the MacArthur study, blood samples were collected by phlebotomists who visited subjects' homes. Although subjects were not required to fast, most of the blood samples were collected in the morning before they had eaten. The time when subjects last ate was recorded. Blood samples were collected for blood chemistry, blood cell counts, and for long-term storage of plasma and blood cells. Blood samples for long-term storage of plasma were collected in lavender top vacutainer tubes (containing ethylenediaminetetraacetic acid), placed immediately in cooler boxes containing blue ice, and returned to the medical centers. Blood was centrifuged immediately upon return at 1500 $g$ for 10 minutes. Plasma was transferred into cryotubes and kept frozen at $-80^{\circ} \mathrm{C}$ until ready for assay. Plasma samples from the selected subjects for this study were shipped to the Research Laboratories of the Bronx VA Medical Center while frozen and were assayed for HVA using high-performance liquid chromatography with coulometric detection (Knott 1990). In most cases enough plasma was also available to assay 3-methoxy4-hydroxyphenylglycol (MHPG) using high-performance liquid chromatography with electrochemical detection (Yang 1988). In our laboratory, the intra- and interassay coefficients of variation for HVA have been estimated to be $2.4 \%$ and $6.0 \%$, respectively, and for MHPG $5.2 \%$ and $13 \%$, respectively.

The motor and similarities task performances had been measured as follows.

Hand-Signature Time. Subjects were seated at a table with a blank piece of paper and a pencil in front of them. Subjects were asked to pick up the pencil, sign their name on the paper, and then put the pencil back on the table. The stopwatch was started when the subjects picked up the pencil and stopped when they replaced it on the table. If a subject took longer than 30 seconds, the test was discontinued.

Walking Time. Subjects were asked to walk on a marked 10-foot course. They were instructed to walk at their usual pace all the way to the end of the course, turn, and come back. Timing was started when the subjects put their first foot on the beginning mark and stopped when one of their feet crossed the finishing mark.

Similarities Task. Each subject was asked four similarities questions taken from Wechsler (1958). Several prompts were permitted on the first item to help them understand the task and to make clear that even though the two things may be alike in a number of ways, the 
most abstract and general concept is the best answer. They were asked how an orange and a banana were alike. If the subject gave a concrete answer, he/she was given an abstract example. If the subject failed to respond or responded incorrectly, the example of a concrete and an abstract response was given. On the subsequent three similarities items, no further cues were permitted. Each response was rated as 4 if abstract, as 2 if concrete, and as 0 if incorrect.

Statistical Analysis. To investigate general trends in the data without the influence of occasional extreme observations, values outside 3 standard deviations from the means were excluded for all variables of interest. Student's $t$-test was used for the comparisons of group means and Pearson product-moment correlation coefficient was used to assess the hypothesized associations between variables. All $p$ values were two sided.

\section{RESULTS}

From the 141 selected subjects - after removing extreme or missing observations - data were available for plasma HVA from $n=138$, for plasma MHPG from $n=114$, for HST from $n=133$, for walking time from $n=134$, and for similarities scores from $n=141$. Males $(n=55)$ and females $(n=86)$ did not differ in their plasma HVA $($ mean \pm SD males $=17.17 \pm 5.98 \mathrm{ng} / \mathrm{ml}$, females $=$ $17.38 \pm 6.64 \mathrm{ng} / \mathrm{ml}, t=0.19, d f=136, p=\mathrm{ns})$, plasma
MHPG $(t=0.18, d f=112, p=\mathrm{NS}), \operatorname{HST}(t=0.51$, $d f=131, p=\mathrm{ns})$, or similarities scores $(t=0.81, d f=$ $139, p=\mathrm{ns}$ ).

A statistically significant negative correlation was observed between plasma HVA and HST $(r=-0.24$, $d f=128, p<.007)$, as shown in Figure 1. Although plasma HVA may be derived from several sources, a major part is derived from DA metabolism in the noradrenergic (NA) system (Amin 1992). To provide some assessment of the relationship of the NA system to the behavioral measures, the association of HST was also examined with plasma MHPG. Plasma MHPG reflects NA metabolism and can provide an indication of HVA contributions from this source. As shown in Figure 2, HST did not correlate with plasma MHPG $(r=$ $-0.04, d f=107, p=n s$ ), suggesting that the association between plasma HVA and HST was not related to the contributions of HVA from the NA system. Although plasma HVA did not decline significantly with the time of day when blood was collected $(r=0.06$, $p=\mathrm{ns}$ ) in this sample, a significant decrease was noted in plasma HVA with increased time duration between blood sample collection and its preparation $(r=-0.21$, $d f=136, p<.02)$. However, when the effect of these variables was statistically removed, the partial correlation between plasma HVA and HST remained significant (partial $r=-0.23, d f=126, p<.001$ ). The walking time did not correlate with plasma HVA ( $r=$ $-0.08, d f=129, p=n s$ ).

A trend level positive correlation was found be-

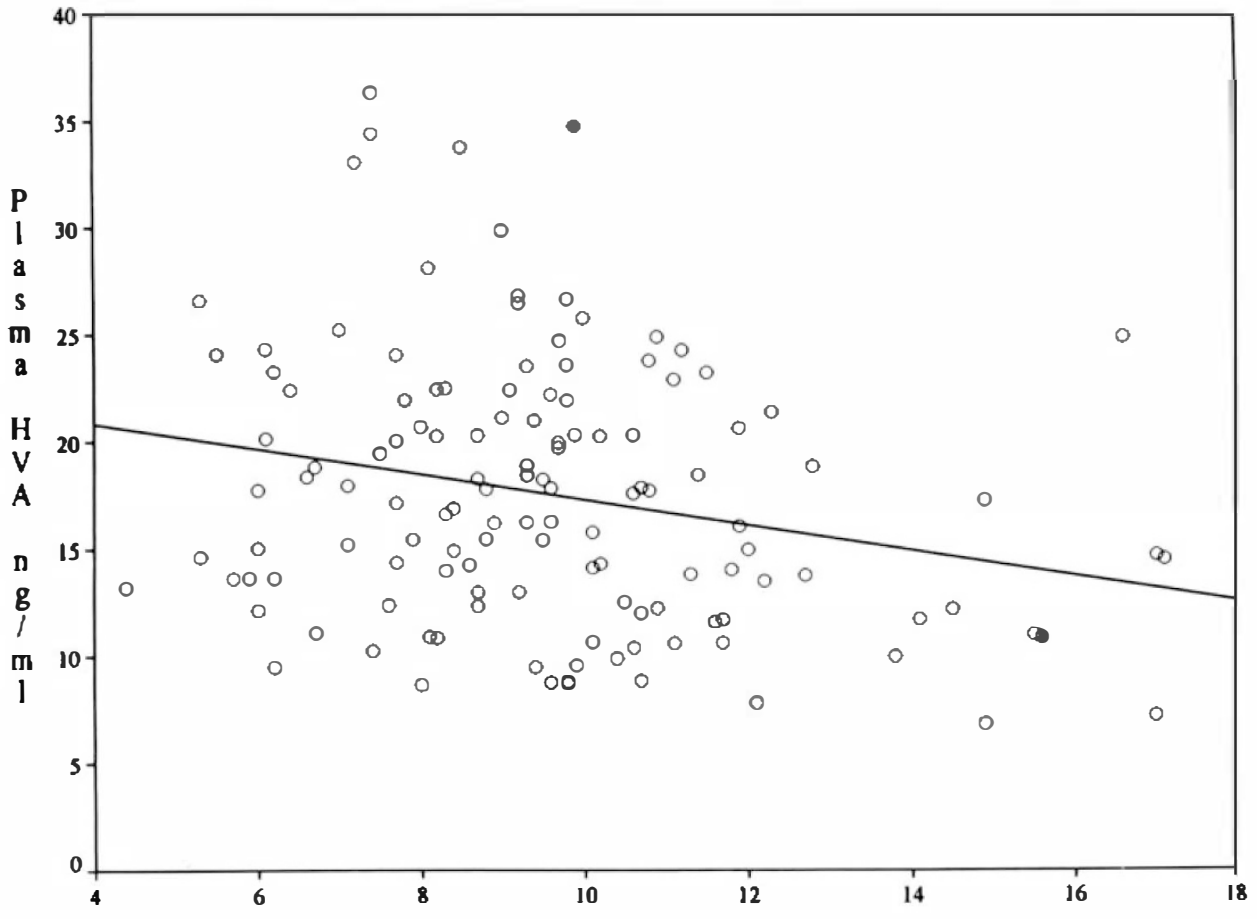

Hand-signature-Time in Seconds
Figure 1. Correlation of plasma HVA and HST in a sample of community-dwelling healthy elderly $(r=-0.24, d f=128$, two-sided $p<.007)$. 
Figure 2. Correlation of plasma MHPG with HST in community-dwelling healthy elderly $(r=-0.04, d f=107$, $p=\mathrm{ns})$.

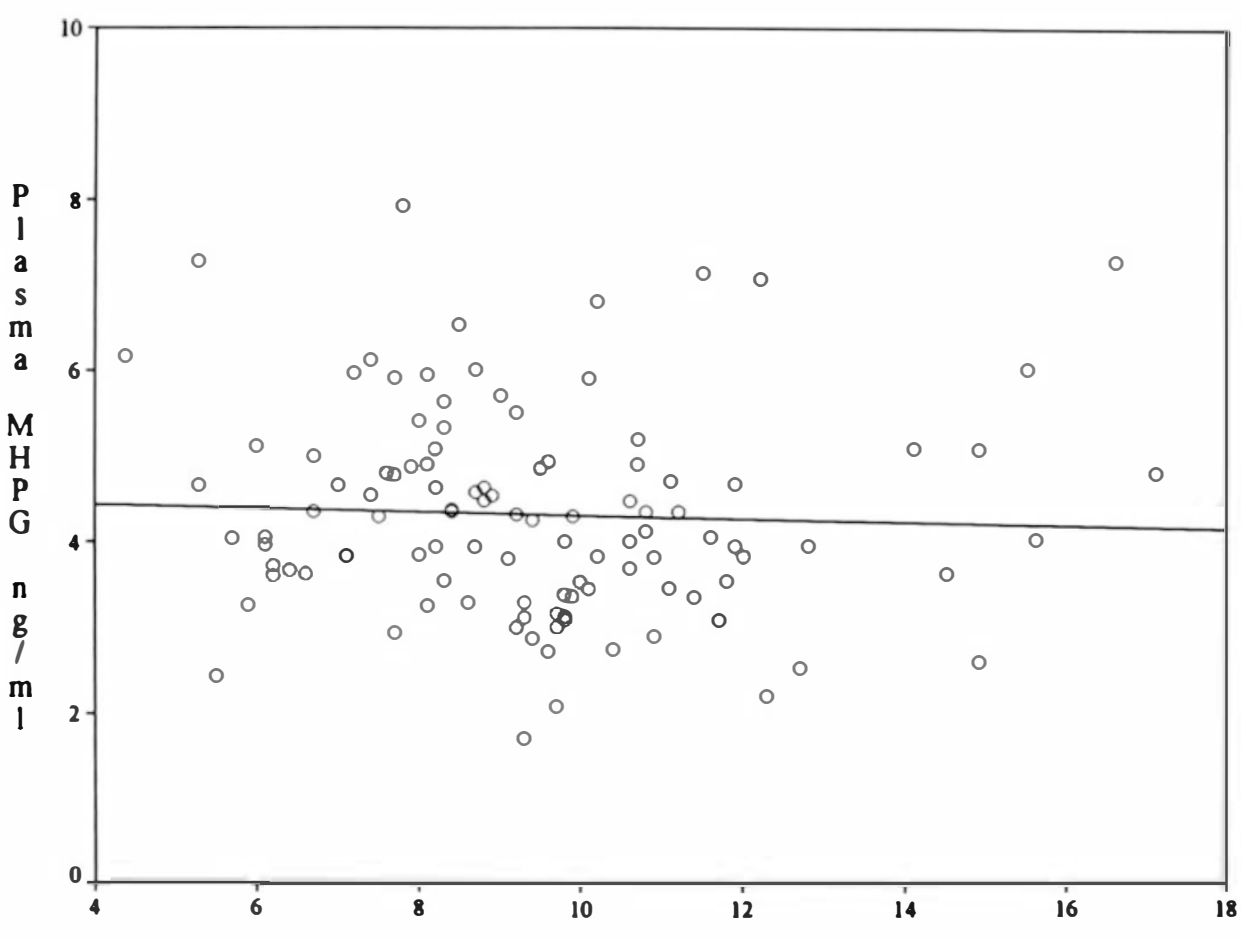

Hand-Signature-Time in Seconds

tween plasma HVA and performance on similarities task $(r=0.15, d f=136, p=.08)$, see Figure 3. However, a statistically significant positive correlation was also found between similarities task performance and plasma MHPG $(r=0.34, d f=112, p=.001)$, as shown in Figure 4. When the effect of plasma MHPG was statistically removed using partial correlation, the trend level association between plasma HVA and similarities task performance was no longer evident (partial $r=$ $0.08, d f=109, p=n s$ ). These results suggest that the
Figure 3. Correlation of plasma HVA with the sum of similarities task scores in a community-dwelling elderly cohort $(r=0.15, d f=136$, two-sided $p=.08)$.

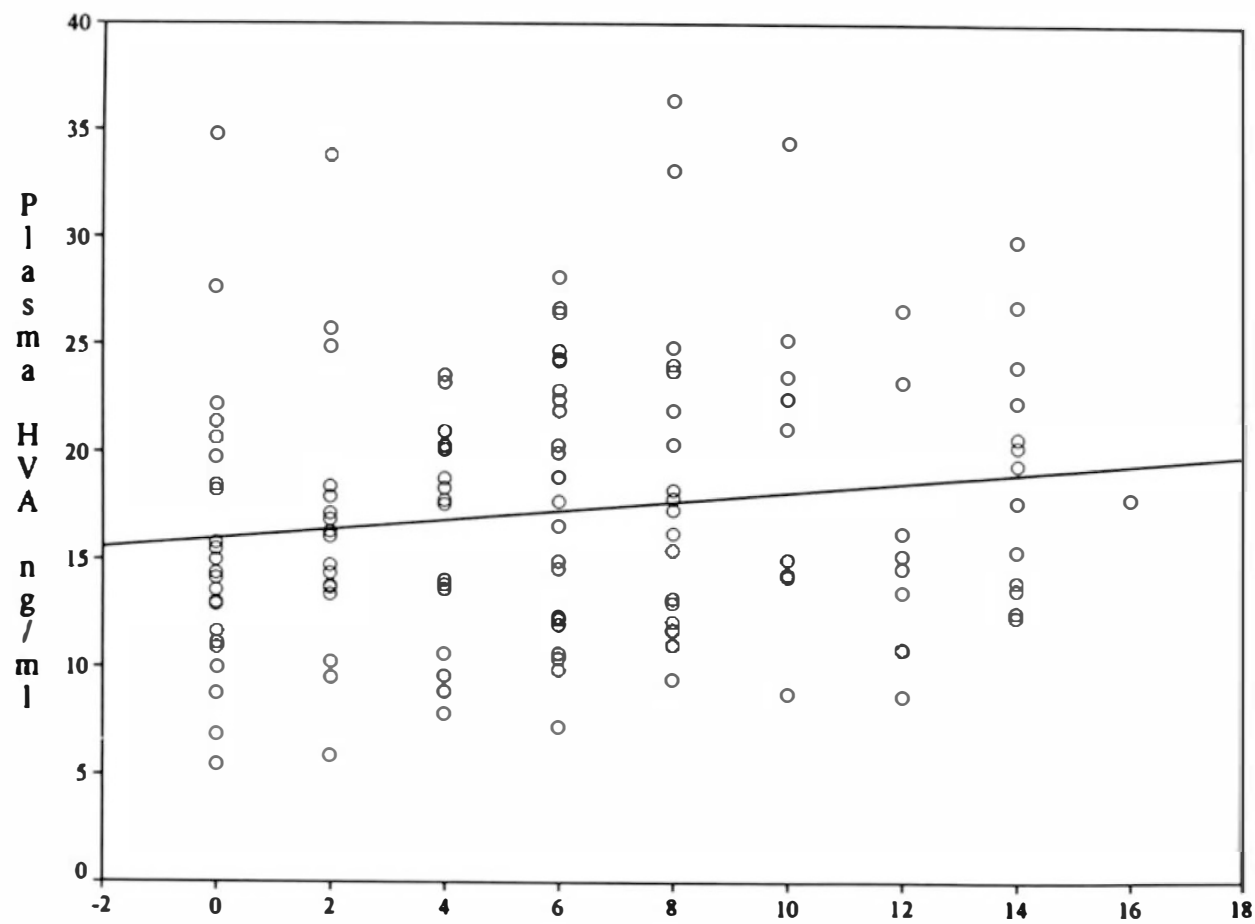

Sum of Similarities Task Scores 


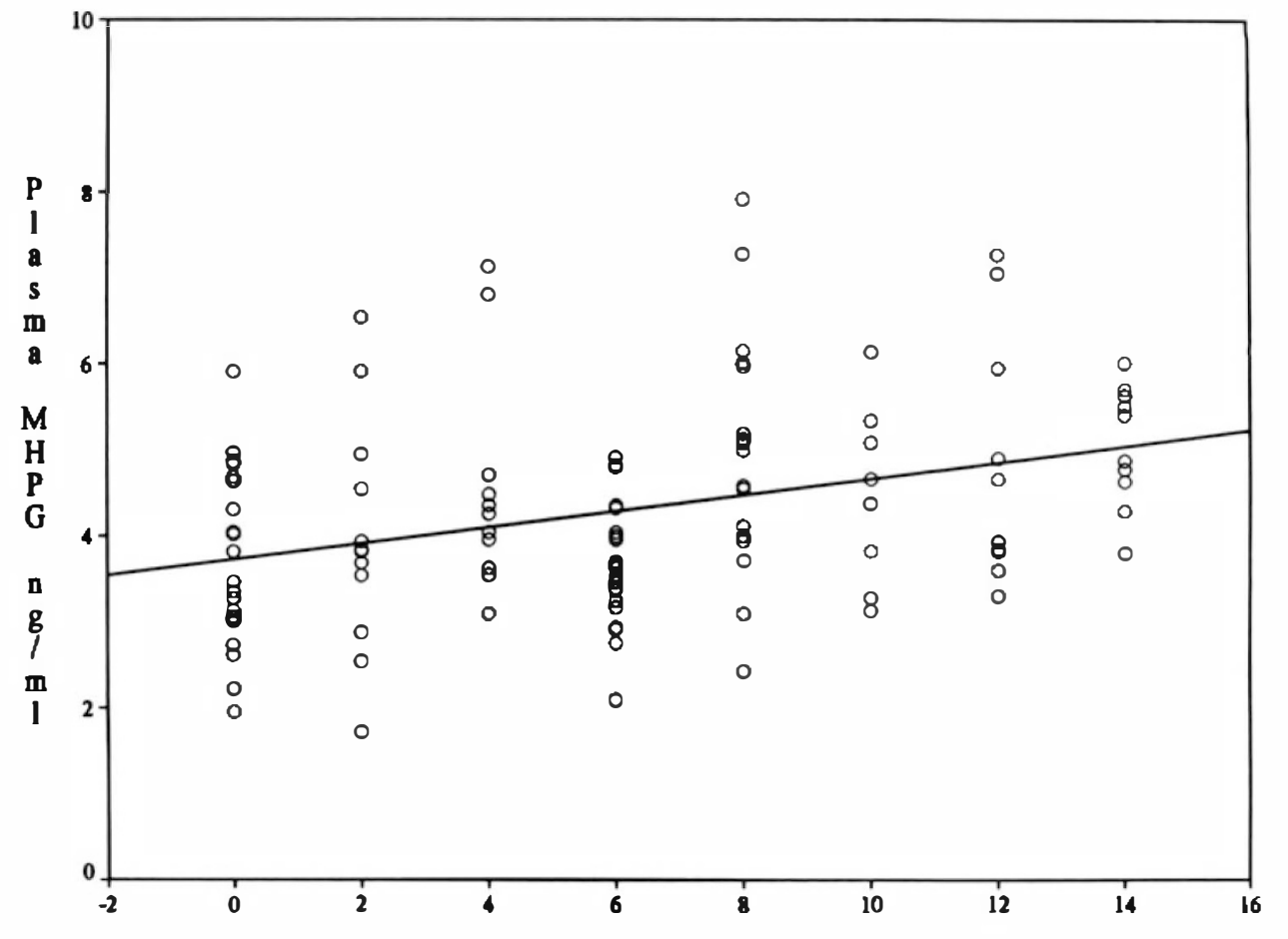

Sum of Scores on Similarities Task
Figure 4. Correlation of plasma MHPG and similarities task performance in communitydwelling healthy elderly $(r=$ $0.34, d f=112$, two-sided $p=$ $.001)$. trend level association between similarities task performance and plasma HVA may be related to HVA contributions from the NA system and probably not to HVA contributions from central DA neurons.

\section{DISCUSSION}

The results indicate that in a community-dwelling healthy elderly population prolonged HST may be associated with decreased plasma HVA. Under fasting conditions, plasma HVA is known to be derived mainly from two sources, that is, central DA neuronal metabolism and NA neuronal metabolism (Amin et al. 1992). The absence of any significant association of HST with the NA metabolite MHPG suggests that the correlation of HST with plasma HVA is not due to the component of HVA derived from NA neurons. Small quantities of HVA may be derived in humans from other sources such as adrenal medulla and SIF cells, but there is no evidence to suggest that their DA metabolisms are related to HST. It is also known that plasma-HVA concentrations are determined not only by its rates of production but also by its renal excretion. In this sample of elderly subjects, plasma HVA significantly correlated positively with serum creatinine and uric acid levels measured from simultaneous blood samples, presumably representing correlates of renal HVA excretion (data to be published in a separate report). How- ever, when the effect of these two variables was statistically removed, the partial correlation between HVA and HST became even stronger (partial $r=-0.30$, two-sided $p<.001$ ), indicating that this association was probably not related to the renal excretion of HVA. Therefore, these results are at least consistent with the view that prolonged HST is associated with lower plasma HVA, possibly reflecting reduced central DA metabolism.

Hand-signature time is a measure of prolonged movement time (bradykinesia), which can be a manifestation of basal ganglia dysfunction. The basal ganglia have been hypothesized to be responsible for the automatic execution of learned motor plans (Marsden 1982), and bradykinesia appears to be one of the most sensitive measures of early Parkinson's disease (Watts 1991). The dramatic reversal of bradykinesia of Parkinson's disease by the DA precursor levodopa in humans and development of bradykinesia along with other parkinsonian symptoms after selective destruction of nigrostriatal DA neurons by 1-methyl-4-phenyl-1,2,3,6tetrahydropyridine in monkeys suggests an association between DA deficiency in basal ganglia and bradykinesia. However, other neurotransmitters can also play a role in basal ganglia function and could conceivably cause the bradykinesia of usual aging. Our results suggest that the bradykinesia of usual aging could be associated with aging-related loss of striatal DA.

In our study, contrary to HST, the walking time did 
not significantly correlate with plasma HVA. It has been suggested that the vulnerability of a given motor function to DA deficiency may be related to its complexity (Tetrud 1991). Handwriting is a complex skill that involves very precise movements of hand and arm muscles and requires good hand-eye coordination, which may be why it is not acquired until the age of approximately 5 to 7 years. Because handwriting is one of the earliest motor skills known to be affected in Parkinson's disease, handwriting changes have been proposed to detect preclinical Parkinson's disease (Tetrud 1991). Our results are consistent with this view and suggest that HST may be an earlier (more sensitive) measure of striatal DA loss compared to walking time. In addition, it is possible that walking time is affected by other aspects of aging that are unrelated to striatal DA loss.

In our study, had plasma-MHPG measurements not been available, the trend level association between plasma HVA and similarities scores could easily have been attributed to central DA metabolism as hypothesized. The data of this study underscore the importance of simultaneous measurement of plasma MHPG in using plasma HVA as an indicator of central DA metabolism to discern relationships with dopaminergic or noradrenergic components of plasma HVA, as proposed previously (Amin et al. 1992). The exact significance of the association between plasma MHPG and similarities task performance in our study is not clear. Because plasma MHPG is derived from both central and peripheral NA metabolism, it is difficult to say from our data whether the association was related to central or peripheral NA metabolism. Further work is needed to clarify this issue.

The modest size of correlation between HST and plasma HVA in our study may have resulted from a number of factors. For example, HST measurement was not controlled for name length. Also, prolonged HST presumably reflected mild to moderately severe deficiency of DA only on one side of the striatum, the one contralateral to the writing hand, which probably was not severe enough to manifest as Parkinson's disease. Furthermore, plasma HVA is derived not only from all of the brain DA neurons but also from the NA neurons of brain and periphery. These factors presumably undermined the strength of the observed correlation. It is possible that if the handwriting test was better standardized (for example, everyone wrote the same sentence) and if a more direct measure of central DA metabolism was available, the association between prolonged handwriting time and central DA metabolism would have been more robust.

Because the elderly subjects in this study were generally healthy and probably free of clinical Parkinson's disease, our results raise the possibility that prolonged HST may be a measure of preclinical striatal DA loss in the elderly. Because of its convenience, HST or handwriting time may be used as part of a screening test battery (Tetrud 1991) to identify elderly individuals who are at risk for developing a more severe striatal DA deficiency and even Parkinson's disease. The need for convenient screening tests to detect DA loss at an early stage has been brought sharply into focus (Langston 1990a) by the advent of "neuroprotective therapy" for the DA system (Langston 1990b) that, if begun early, could possibly delay or even prevent the onset of Parkinson's disease (Tetrud and Langston 1989; Shoulson et al. 1989). A similar strategy has been proposed to delay the age-related decline of striatal DA in Alzheimer's disease patients (Knoll 1992).

\section{ACKNOWLEDGMENT}

This work was supported by the John D. and Catherine T. MacArthur Foundation Research Network on Successful Aging. Authors acknowledge the technical assistance of Donna D. Holland and Phyllis Lam in assaying plasma HVA and MHPG.

\section{REFERENCES}

Amin F, Davidson M, Davis KL (1992): Homovanillic acid measurement in clinical research: A review of methodology. Schizophr Bull 18:123-148

Bacopoulos NG, Hattox SE, Roth RH (1979): 3,4-Dihydroxyphenylacetic acid and homovanillic acid in rat plasma: Possible indicators of central dopaminergic activity. Eur J Pharmacol 56:225-236

Berkman LF, Seeman TE, Albert M, Blazer D, Kahn R, Mohs R, Finch C, Schneider E, Cotman C, McCearn G, Nesselroade J, Featherman D, Garmezy N, McKhann G, Prager D, Rowe J (1994): Successful, usual and impaired functioning: Findings from the MacArthur Foundation network on successful aging multi-site study of community-dwelling elderly. J Clin Epidemiol (In press)

Carlsson A, Winblad B (1976): Influence of age and time interval between death and autopsy on dopamine and 3-methoxytyramine levels in human basal ganglia. J Neural Transm 38:271-276

Cornoni-Huntley J, Brock DB, Ostfeld AM, Taylor JO, Wallace RB (1986): Established populations for the epidemiologic studies of the elderly: Resource data book. NIH Publication No. 86-2443, NIH Bethesda, MD

Hornykiewicz O, Pifl C, Schingnitz G, Kish SJ (1988): The cause of Parkinson's disease: MPTP, aging and striatal dopamineloss. In Nappi G (ed), Neurodegenerative Disorders: The Role Played Endotoxins and Xenobiotics. New York, Raven Press, pp 73-80

Inouye SK, Albert MS, Mohs RC, Sun K, Berkman LF (1994): Cognitive performance in a high-functioning communitydwelling elderly population. J Gerontol (In press)

Kendler KS, Davis KL (1984): Acute and chronic effects of neuroleptic drugs on plasma and brain homovanillic acid in the rat. Psychiatry Res 13:51-58 
Kendler KS, Hsieh JY-K, Davis KL (1982): Studies of plasma homovanillic acid as an index of brain dopamine function. Psychopharmacol Bull 18:152-155.

Knoll J (1992): ( - )Deprenyl-medication: A strategy to modulate the age-related decline of the striatal dopaminergic system. J Am Geriatr Soc 40:839-847

Knott PJ, Yang RK, Cheng $\mathrm{H}$, Warne P, Haroutunian V, Davidson M, Davis KL (1992): An improved, sensitive method for measurement of plasma homovanillic acid by HPLC with coulometric detection. Presented at 14th International Symposium on Column Liquid Chromatography, May 20-25, Boston, MA

Konicki PE, Owen RR, Litman RE, Pickar D (1991): The acute effects of central- and peripheral-acting dopamine antagonists on plasma HVA in schizophrenic patients. Life Sci 48:1411-1416

Langston JW (1990a): Predicting Parkinson's disease. Neurology 40(Suppl 3):70-74

Langston JW (1990b): Selegiline as neuroprotective therapy in Parkinson's disease: Concepts and controversies. Neurology 40(Suppl 3):61-66

Loranger AW, Goodell H, McDowell F, Lee JE, Sweet RD (1972): Intellectual impairment in Parkinson's syndrome. Brain 95:405-412

McFie J (1975): Assessment of Organic Intellectual Impairment. New York, Academic Press

McGeer PL, McGeer EG, Suzuki JS (1977): Aging and extrapyramidal function. Arch Neurol 34:33-35

Shoulson I, Fahn S, Oakes D, et al. (1989): Effect of deprenyl on the progression of disability in early Parkinson's dis- ease. The Parkinson's Study Group. N Engl J Med 321: 1364-1371

Spirduso WW (1975): Reaction and movement time as a function of age and physical activity level. J Gerontol 30: 435-440

Spirduso WW, Clifford P (1978): Replication of age and physical activity effects on reaction and movement time. J Gerontol 33:26-30

Stern Y, Langston JW (1985): Intellectual changes in patients with MPTP-induced parkinsonism. Neurology 35: 15061509

Tetrud JW (1991): Preclinical Parkinson's disease: Detection of motor and non-motor manifestations. Neurology 41 (Suppl 2): 69-72

Tetrud JW, Langston JW (1989): The effect of deprenyl (selegiline) on the natural history of Parkinson's disease. Science 245:519-522

Wallin A, Gottfries CG (1990): Biochemical substrates in normal aging and Alzheimer's disease. Pharmacopsychiatry 23(Suppl 2): 37-43

Watts RL, Mandir AS, Ahn KJ, Juncos JL, Zakers GO, Freeman A (1991): Electrophysiologic analysis of early Parkinson's disease. Neurology 41 (Suppl 2):44-48

Wechsler D (1958): The Measurement and Appraisal of Adult Intelligence. Baltimore, Williams \& Wilkins

Yang RK, Campbell G, Cheng H, Tsuboyama GK, Davis KL (1988): Highly sensitive and simple method for determination of free 3-methoxy-4-hydroxyphenylglycol in plasma by high-performance liquid chromatography using a SepPak alumina B cartridge. J Liquid Chromatogr 11:32233231 